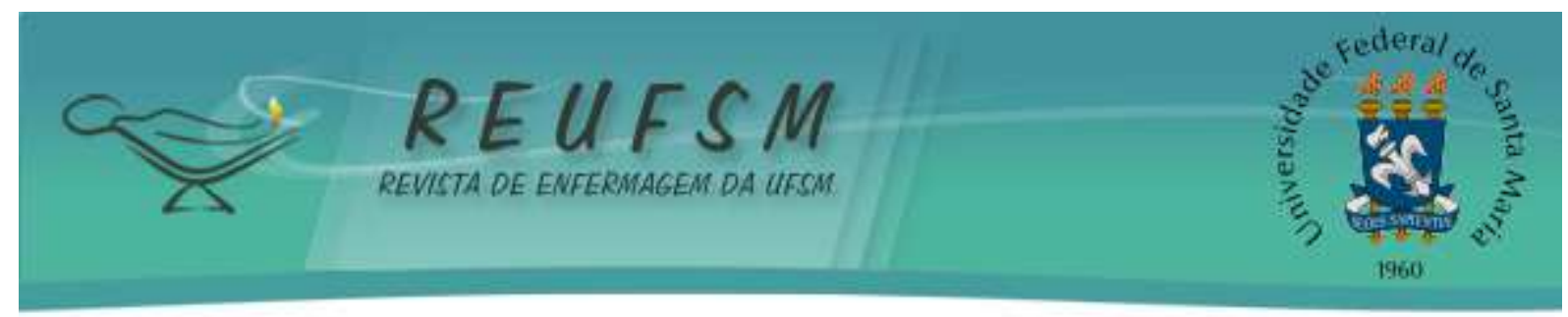

ARTIGO ORIGINAL

\title{
ARTICULAÇÃO ENTRE SISTEMATIZAÇÃO DE ENFERMAGEM E PROCEDIMENTOS OPERACIONAIS: PERSPECTIVA DOS ENFERMEIROS DOS BANCOS DE LEITE
}

ARTICULATION BETWEEN NURSING SYSTEMS AND OPERATIONAL PROCEDURES: NURSING PERSPECTIVES OF MILK BANKS

\section{ARTICULACIÓN ENTRE SISTEMATIZACIÓN DE ENFERMERÍA Y PROCEDIMIENTOS OPERACIONALES: PERSPECTIVA DE ENFERMEROS EN BANCOS DE LECHE}

\author{
Giovanna Rosario Soanno Marchiori ${ }^{1}$ \\ Valdecyr Herdy Alves ${ }^{2}$ \\ Diego Pereira Rodrigues ${ }^{3}$ \\ Adriana Duarte Gabriel ${ }^{4}$ \\ Marcia Vieira dos Santos 5 \\ Maria Bertilla Lutterbach Riker Branco ${ }^{6}$
}

Doi: $10.5902 / 2179769225701$

RESUMO: Objetivo: compreender as facetas de articulação da Sistematização da Assistência de Enfermagem com as normativas utilizadas pelos enfermeiros do Banco de Leite Humano. Método: estudo descritivo de abordagem qualitativa, realizado de maio a novembro de 2014, nos sete Bancos de Leite do Estado do Espírito Santo, onde foram entrevistadas nove enfermeiras. Os dados foram submetidos à análise de conteúdo na modalidade temática. Resultados: após a análise dos dados, emergiram as categorias: conhecimento dos enfermeiros sobre a Sistematização da Assistência de Enfermagem para sua aplicabilidade; A Sistematização da Assistência de Enfermagem e os procedimentos operacionais padrão em banco de leite humano: uma articulação do processo de enfermagem. Conclusão: verificou-se que há necessidade de se implementar a Sistematização da Assistência de Enfermagem nesse espaço especializado e a necessidade de se elaborar estratégias as quais articulem a Sistematização da Assistência de Enfermagem e Procedimentos Operacionais nos Bancos de Leite Humano.

Descritores: Processos de enfermagem; Cuidados de enfermagem; Bancos de leite; Normas técnicas.

\footnotetext{
${ }^{1}$ Enfermeira. Mestre em Saúde Materno Infantil, Universidade Federal Fluminense. Professora da Faculdade Novo Milênio. Espirito Santo, Brasil. E-mail: giovannasoanno@gmail.com

${ }^{2}$ Enfermeiro. Doutor em Enfermagem. Professor Titular do Departamento Materno-Infantil e Psiquiátrico da Escola de Enfermagem Aurora de Afonso Costa da Universidade Federal Fluminense. Rio de Janeiro, Brasil. Email: herdyalves@yahoo.com.br

3 Enfermeiro. Mestre em Enfermagem. Universidade Federal Fluminense. Vice-presidente da Associação Brasileira de Obstetrizes e Enfermeiros Obstetras do Estado do Rio de Janeiro. Rio de Janeiro, Brasil. E-mail: diego.pereira.rodrigues@gmail.com

${ }^{4}$ Enfermeira. Mestre em Saúde Materno Infantil, Universidade Federal Fluminense. Rio de Janeiro, Brasil. Email: adriannadg@gmail.com

5 Enfermeira. Mestre em Saúde Materno Infantil. Enfermeira da Secretaria de Estado de Administração Penitenciária. Rio de Janeiro, Brasil. E-mail: enfa.marcia@oi.com.br

${ }^{6}$ Enfermeira. Mestre em Saúde Materno Infantil. Enfermeira do Banco de Leite Humano do Hospital Universitário Antônio Pedro, Universidade Federal Fluminense. Rio de Janeiro, Brasil. E-mail: bertillariker@yahoo.com.br
} 


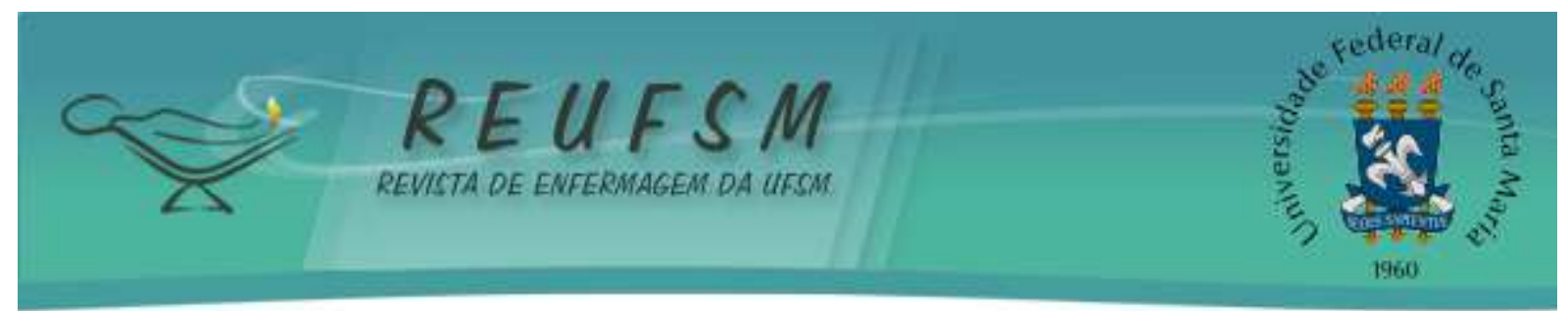

ABSTRACT: Aim: to understand the articulation facets of Nursing Care Systematization with the regulations used by nurses of the Human Milk Bank. Method: a descriptive study of a qualitative approach, carried out from May to November 2014, in the seven milk banks of the State of Espirito Santo, where nine nurses were interviewed. The data were submitted to content analysis in the thematic modality. Results: after data analysis, the following categories emerged: Nurses' knowledge about Nursing Care Systematization for its applicability; The Nursing Care Systematization and the standard operating procedures in the human milk bank: an articulation of the nursing process. Conclusion: the need to implement the Nursing Care Systematization in this specialized location and the need to elaborate strategies that articulate the Nursing Care Systematization and Operational Procedures in Human Milk Banks were confirmed.

Descriptors: Nursing process; Nursing care; Milk banks; Technical standards.

RESUMEN: Objetivo: entender las particularidades de la articulación entre la Sistematización de la Asistencia de Enfermería con las normativas utilizadas por los enfermeros del Banco de Leche Humana. Método: estudio descriptivo con enfoque cualitativo, realizado de mayo a noviembre de 2014, en los siete bancos de leche en el Estado de Espírito Santo, en los cuales se entrevistó nueve enfermeros. Los datos fueron sometidos a análisis de contenido temático. Resultados: surgieron las categorías: Conocimiento de los enfermeros sobre la Sistematización de la Asistencia de Enfermería para su aplicabilidad; La Sistematización de la Asistencia de Enfermería y los procedimientos operacionales estándar en banco de leche humana: una articulación del proceso de enfermería. Conclusión: la necesidad de implementar la sistematización de la enfermería en Bancos de Leche Humana espacio y desarrollar estrategias que articulan la sistematización de enfermería y los procedimientos operativos en este espacio.

Descriptores: Procesos de enfermería; Atención de enfermería; Bancos de leche; Normas técnicas.

\section{INTRODUÇÃO}

O Banco de Leite Humano (BLH), segundo a Resolução da Diretoria Colegiada (RDC) 171 é um serviço especializado de apoio, promoção e proteção ao aleitamento materno. Essa Resolução enfatiza as atividades de coleta, processamento e controle de qualidade do leite humano ordenhado, para distribuição aos bebês prematuros e baixo peso, internados em Unidade de Terapia Intensiva Neonatal (UTIN). ${ }^{1}$

Nas questões de atenção à saúde materno-infantil, novas propostas e necessidades surgem com a consolidação das ações e serviços prestados nos BLH de todo o Brasil. É o caso da Portaria GM/MS nº 698/2002, que define a estrutura e as normas de atuação e funcionamento dos BLH; da Resolução da Diretoria Colegiada - RDC n 171/2006 da Agência Nacional de Vigilância Sanitária (ANVISA), que dispõe sobre o regulamento técnico para o seu funcionamento; e da Portaria GM/MS n ${ }^{\circ}$ 2.193/2006, documento que define a estrutura e funcionamento dos BLH, revogando a Portaria MS nº 322/1988, um importante marco histórico. ${ }^{2}$ 


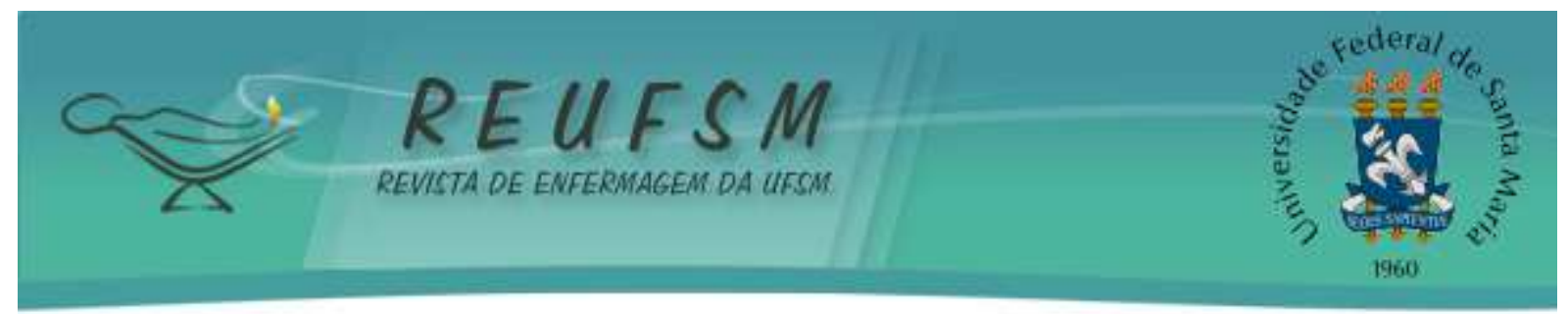

Nesse espaço especializado, os profissionais que nele atuam, dentre eles o enfermeiro, devem observar os procedimentos operacionais direcionados ao BLH, que servem como base no atendimento ao cliente/paciente. Como orientador normativo, as normas técnicas e o Manual do BLH estabelece os requisitos mínimos para o funcionamento, promoção e controle de riscos. ${ }^{2-3}$

Em consonância com o Artigo 11 da Lei 7498/86 do Exercício Profissional da Enfermagem, cabe ao enfermeiro exercer todas as atividades de Enfermagem e, privativamente, a direção do órgão de enfermagem integrante da estrutura básica da instituição de saúde, chefia de serviço e de unidade de saúde; planejamento, organização, coordenação, execução e avaliação da assistência de enfermagem; consulta de enfermagem; cuidados diretos de enfermagem a pacientes graves com risco de vida; cuidados de enfermagem de maior complexidade técnica e que exijam conhecimento de base científica, capacidade de tomar decisões rápidas, atuar como coordenador, supervisor ou assistencial nos BLH. ${ }^{2,4}$

A Sistematização da Assistência de Enfermagem (SAE) surgiu quando a Enfermagem começou a sistematizar as suas ações com o intuito de integrar o seu conhecimento teórico à prática assistencial e, assim, construir um corpo de conhecimento autônomo na busca de consolidar a profissão enquanto ciência. Com o advir de um saber científico próprio, a Enfermagem prima pela indissociabilidade entre o pensar, o ser e o agir, o que retira as amarras que ainda a subjugam, bem como qualifica a prática assistencial. ${ }^{5}$

Portanto, a SAE é conceituada como método de prestação de cuidados para a obtenção de resultados satisfatórios na implementação da assistência, cuja utilização requer o pensamento crítico do profissional, que deve estar focado nos objetivos e nos resultados. Logo, é um modo de exercer a profissão de enfermeiro com autonomia baseada nos conhecimentos técnicos e científicos, nos quais a categoria vem embasando e desenvolvendo seus saberes nas últimas décadas, assim aumentando a visibilidade e o reconhecimento profissional. ${ }^{6-7}$

Nesse sentido, de acordo com a Resolução COFEN 358/2009, ${ }^{8}$ o Processo de Enfermagem (PE) é a dinâmica das ações sistematizadas e inter-relacionadas que viabiliza a organização da assistência de enfermagem a partir de uma abordagem ética e humanizada, dirigida à resolução de problemas que visa atender às necessidades de cuidados de saúde de uma pessoa e indica uma direção para possíveis intervenções. ${ }^{9-10}$

A articulação com os procedimentos operacionais padrão (POP) indica a possibilidade de sustentar o processo de enfermagem, uma vez que garante a efetividade no trabalho, pode 


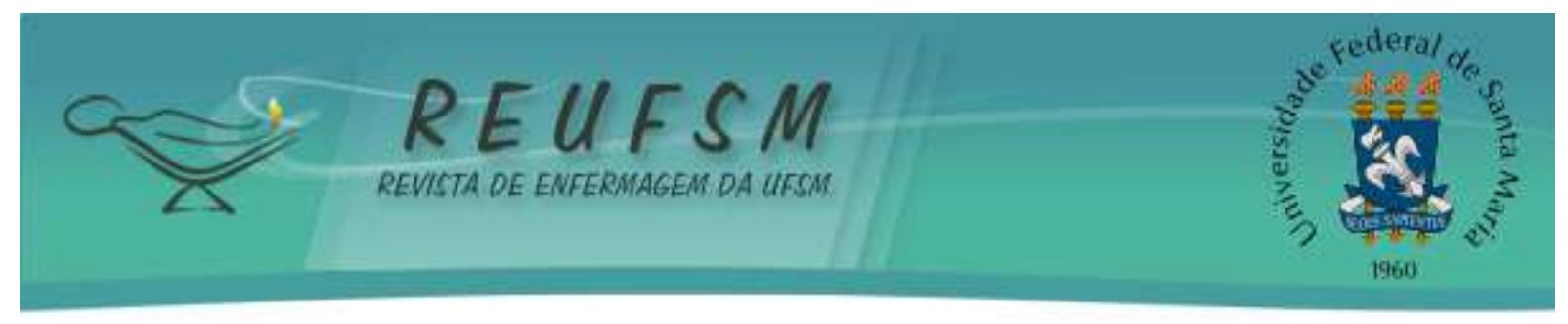

levar a visão global da atenção ao binômio, favorecendo a continuação da assistência e direcionando-a através do embasamento científico. ${ }^{11}$

A SAE, além de trazer benefícios para as famílias, as instituições e a sociedade repercute favoravelmente na atuação do enfermeiro no BLH, cujo processo de cuidado é direcionado às mulheres gestantes, ao parto-nascimento e ao puerpério. Há, também, contribuição para a qualidade de vida quando, por exemplo, as intervenções de enfermagem garantem, junto ao foco do cuidado, menores riscos para a lactação decorrentes dos transtornos da amamentação, tendo em vista o alcance do objetivo principal do BLH que é o apoio, a promoção e a proteção ao aleitamento.,

Diante do exposto emergiu a seguinte questão de pesquisa: de que forma estão articulados a SAE e as normativas utilizadas pelo enfermeiro no BLH?

O estudo é pertinente para o contexto nacional e pelo fato da cooperação internacional em saúde entre Brasil e os membros da Comunidade dos Países de Língua Portuguesa. ${ }^{12} \mathrm{Na}$ atualidade, nas Redes de BLH, sejam nacionais ou internacionais, a SAE não está implementada. No campo internacional, autores apontam as mesmas dificuldades em se utilizar o PE, como um instrumento da SAE, sinalizando motivos para isso: falta de conhecimento sobre o PE, maior o número de pacientes atendidos, maior necessidade de adequação das tecnologias de assistência e recursos humanos capacitados, educação continuada, mobilização da equipe e apoio da gestão. ${ }^{13-14}$

Para tanto, foi estabelecido como objetivo deste artigo compreender as facetas de articulação da Sistematização da Assistência de Enfermagem com as normativas utilizadas pelos enfermeiros do Banco de Leite Humano. As normativas do BLH utilizadas pelo enfermeiro que foram objeto de análise são: Portaria MS no 812; RDC ANVISA n 171/2006; Portaria GM/MS no 2.193/2006; Portaria MS no 2193/2006; A Portaria n 930/2012; Portaria, de n 961/2013; Manual ANVISA intitulado "Banco de Leite Humano: Funcionamento, Prevenção e Controle de Riscos".

\section{MÉTODO}

Trata-se de estudo descritivo com abordagem qualitativa ${ }^{15-16}$ nos sete Bancos de Leite Humano de hospitais do Estado do Espírito Santo, sendo cinco públicos e dois filantrópicos.

Nesta pesquisa, foram convidadas para participar do estudo onze enfermeiras que atuavam nos BLH dessas instituições, uma vez que correspondia ao número total de 


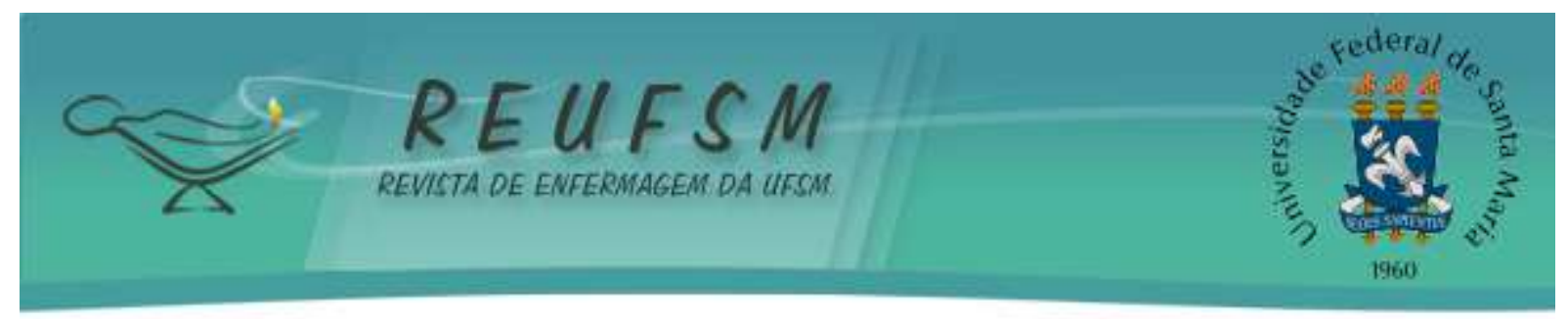

profissionais atuantes nos BLH do Estado do Espírito Santo. Os critérios inclusão para seleção das participantes foram: ter participado do curso de capacitação em aleitamento materno, ter no mínimo seis meses de experiência no setor e estar atuante na coordenação, supervisão ou assistência do BLH. Quanto aos critérios de exclusão: as enfermeiras que estivessem fora da escala de serviço por gozo de férias, licença maternidade, prêmio ou doença, no momento da coleta de dados. Duas enfermeiras foram excluídas do estudo, sendo: uma enfermeira em gozo de férias e outra admitida há uma semana no setor.

A coleta de dados foi realizada no período de maio à novembro de 2014, com a aplicação da técnica de entrevista com roteiro semiestruturado, com perguntas abertas e fechadas referentes ao conhecimento dos enfermeiros em relação à SAE. Os depoimentos foram gravados em aparelho digital e transcritos na íntegra, com autorização prévia de cada participante. As variáveis sociodemográficas e profissionais foram direcionadas por roteiro semiestruturado, que contemplou uma organização que permitiu a identificação do enfermeiro por sexo, idade, tempo de atuação no BLH, conhecimento da SAE, etapas do PE e a utilização do POP.

Os dados foram submetidos à análise de conteúdo na modalidade temática, ${ }^{16}$ que ocorreu em diferentes fases: pré análise; exploração do material; tratamento dos resultados, inferência e a interpretação. Também assegurou-se o anonimato e o sigilo das informações às entrevistadas, confirmado com a utilização de um código alfanumérico (E1...E9), assim viabilizando a aplicação do instrumento de coleta de dados.

O estudo foi aprovado pelo Comitê de Ética em Pesquisa (CEP) da Faculdade de Medicina do Hospital Universitário Antônio Pedro (HUAP) da Universidade Federal Fluminense (UFF), CAAE: 27224214.9.0000.5243 sob o parecer 978444 de 06/03/2015, em conformidade com a Resolução n ${ }^{\circ}$ 466/2012 do Conselho Nacional de Saúde (CNS), a elaboração, esclarecimento dos objetivos do estudo, metodologia, benefícios do estudo e posterior assinatura do Termo de Consentimento Livre e Esclarecido (TCLE) pelas participantes.

\section{RESULTADOS}

As categorias que emergiram das falas das entrevistadas foram "o conhecimento dos enfermeiros sobre a Sistematização da Assistência de Enfermagem para sua aplicabilidade" e "a Sistematização da Assistência de Enfermagem e os Procedimentos Operacionais Padrão em Banco de Leite Humano: uma articulação do processo de enfermagem”. 


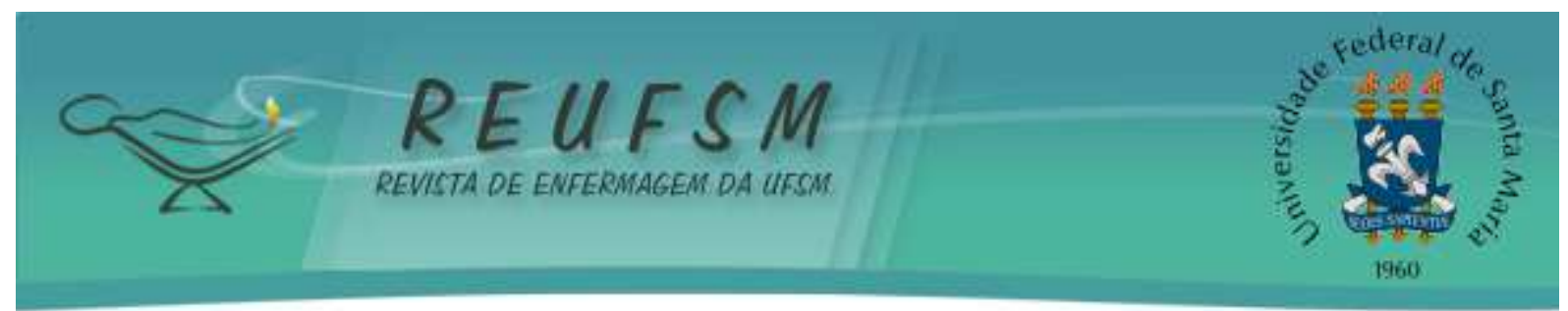

Registramos aqui que participaram do estudo nove enfermeiras, do sexo feminino, a faixa etária apresentou variação entre 30 e 51 anos. Quanto ao tempo de atuação no BLH houve variação de sete meses a vinte e um anos.

\section{O conhecimento dos enfermeiros sobre a Sistematização da Assistência de Enfermagem para sua aplicabilidade}

A SAE capacita os enfermeiros a se comunicarem com os profissionais de todas as especialidades clínicas e setores, visto que este método possui uma linguagem que lhes é comum e familiar.

Quando foram indagadas sobre "o que entendiam sobre SAE", não houve uma resposta que refletisse seu significado teórico, mas sim, uma consciência da importância da prática, como exemplificado nos depoimentos a seguir:

olha, eu entendo que você vai trabalhar com o cliente como um todo. Você não vai olhar diretamente ou só a queixa naquele momento. Quando você faz anamnese, você já percebe na fala dele. (E1)

o que você faz, a orientação, a prescrição e atuação. E também não adianta você só prescrever, não atuar e não observar quem está atendendo. (E2)

Outras participantes reconheceram a SAE como um acompanhamento do processo de atendimento/atenção à nutriz e ao concepto, especialmente porque esta metodologia configura-se como processo dinamizador da assistência. Seguem depoimentos a respeito:

é uma coisa complexa [pensativa], mas enfim, eu vejo a sistematização, na realidade, como um ganho para a enfermagem. E chega uma hora que fica uma coisa muito rotineira. Então, essa questão é de atentar mais às necessidades mesmo de cada Ser humano, em focar mesmo nessa questão de orientação mais individualizada, cuidado mais individualizado. (E3)

eu acho que SAE para mim, no meu dia a dia, é um acompanhamento mais criterioso e sequencial do paciente. (E5)

Sabe-se que o (re)conhecimento das enfermeiras acerca de SAE e a sua aplicabilidade são para além do espaço do Banco de Leite Humano e envolve questões para além do querer única e exclusivamente do profissional tendo haver, principalmente, com questões gerenciais como recursos humanos. Conforme as falas analisadas, há indicativos de um saber teórico consolidado sobre a SAE. Contudo, o não (re)conhecimento ou consciência de que as ações da enfermagem necessitam estar pautados em métodos científicos pode significar a não 


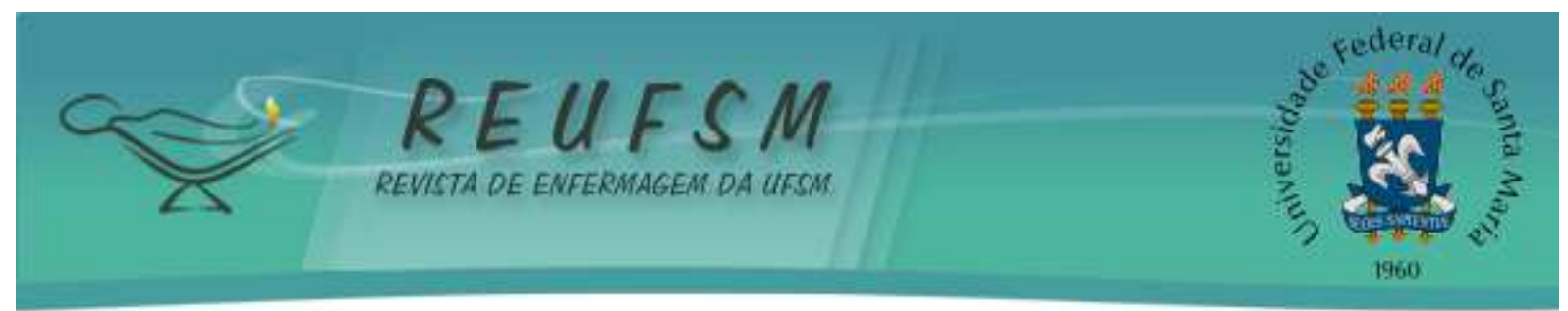

realização da SAE, bem como compromete a consolidação do Processo de Enfermagem, seu instrumento metodológico.

\section{A Sistematização da Assistência de Enfermagem e os procedimentos operacionais} padrão em Banco de Leite Humano: uma articulação do processo de enfermagem

As práticas de enfermagem seguem qualificando a atenção aos pacientes que procuram o BLH. Entretanto, frente às demandas da profissão e normas técnicas que regem as ações da enfermagem, a SAE necessita ser observada e exercida em qualquer ambiente da saúde. Ainda, constatou-se que as participantes do estudo não conseguiam articular a SAE com os POP que estão no Manual do BLH, isto se torna de fato um problema perceptível, de acordo com os depoimentos a seguir:

a gente está pegando linha por linha do manual e resolvendo as coisas que eram mais gritantes, de documentação de registro para poder organizar e aos poucos eu estou seguindo à risca. (E6)

isso aqui pra mim é a Bíblia [referindo-se ao Manual técnico do BLH] que a gente tem. (E7)

Nessa visão, a ênfase atribuída ao documento aparenta se sobrepor aos conhecimentos da SAE. Sendo assim, subentende-se que há um compromisso com as normas técnicas e, possivelmente, uma exclusão do método científico ou a sua invisibilização.

Então, questiona-se até que ponto a falta de articulação entre a SAE e os POP nos BLH interfere na qualidade do serviço prestado à população nesse espaço especializado. As falas analisadas indicam o manual como instrumento norteador, indicando a não efetivação do Processo de Enfermagem:

é o que eu te falei, a gente está revisando e vai formatar tudo para colocar tudo no sistema. O que nós estamos utilizando hoje no dia a dia, no setor, é o manual mesmo da ANVISA. Nós nos baseamos nele hoje e a gente se baseou também para montar os POP. (E3)

a gente utiliza até mesmo porque o nosso POP é muito vinculado ao manual para BLH. Então, ali já dá uma base total do acompanhamento dessas mães e também da parte técnica do Banco de Leite. Então até as funcionárias que entram novas precisam conhecer para que possam colocar em prática. O POP dá uma segurança do que você está fazendo. (E5)

Quando confrontadas com situações que não constam no Manual Técnico, as participantes revelaram que recorrem a outros profissionais para a solução de problemas e 


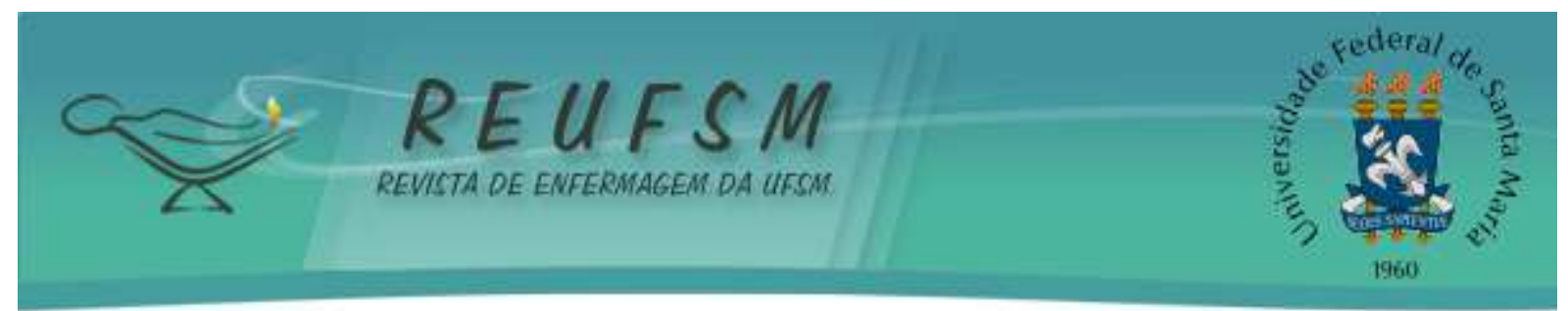

atenção ao paciente. Considerando-se que há uma equipe multiprofissional atuando nesse espaço, uma demanda específica exige a mobilização de fontes externas:

elas são muito assim, agora tem como [...] eu estou aqui direto, assim elas respeitam muito o enfermeiro, esta questão de ter aqui o enfermeiro e pediatra, [...] tem coisas que elas passam tudo para mim, então a gente consegue elaborar uma situação e desenvolver a ação como equipe. (E1)

ás vezes acontece, quando a gente sabe a solução a gente procura resolver, caso contrário a gente, às vezes, recorre à doutora ou ao próprio hospital. (E6)

Também foi relatado que, em caso de dúvidas ou em caso não previstos, recorre-se à Rede BLH-BR. Quando não há uma prescrição prevista no Manual, exige-se das participantes um preparo para atuar e buscar embasamento teórico, a fim de resolver a demanda e atender o paciente. $\mathrm{Na}$ fala das entrevistadas há o reconhecimento sobre o Processo de Enfermagem e a aplicação de conhecimentos técnicos da profissão. O trabalho e a articulação da equipe, de certa forma, atende à demanda do BLH, como pode ser constatado:

quando existe alguma dúvida e não tem orientação neste manual, [...], elas vêm ao enfermeiro, quando tem dúvida, recorrem a mim. (E3) para lidar com situações, por exemplo, a mulher chega falando que não consegue dar a mama. Não é nada disso, mas o leite não desce, às vezes é emocional, e ai? Não está escrito isso no manual. Ai a gente mesmo assim põe essa mãe na sala, e como o número de atendimentos é muito grande, nós vamos dar atenção para essa mãe, vai ouvir, muitas vezes, essa mãe está sobrecarregada. (E9)

Há uma dicotomia que se percebe nas falas das enfermeiras, frente às prescrições no manual técnico. Se não está prescrito, automaticamente é exigida dessa profissional a capacidade de sistematizar a assistência de enfermagem para operacionalizar o processo de enfermagem.

\section{DISCUSSÃO}

As ações do profissional enfermeiro necessitam estar pautados num valor de suma importância que é o conhecimento. Entende-se que para a tomada de decisões, este profissional precisa, necessariamente, ter a segurança para prática cotidiana, e utilizar métodos científicos para sua autonomia e reconhecimento profissional, onde quer que atue. A carência de conhecimento ou a plena consciência a respeito da SAE mostra-se claramente nos depoimentos das enfermeiras do BLH.

Dessa forma, a SAE é definida como um fenômeno interativo e complexo que abrange o saber, o fazer e o legislar, sendo o enfermeiro reconhecido como o profissional articulador 


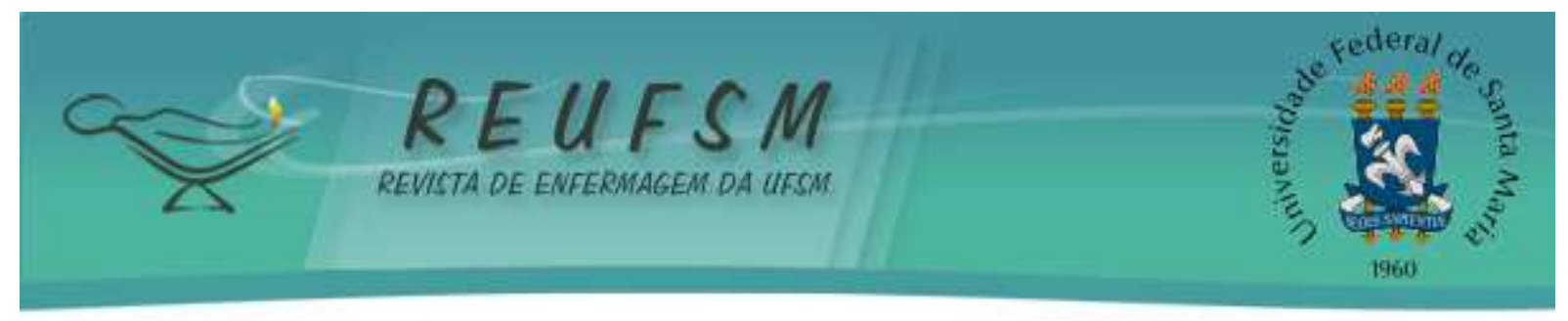

desses diferentes saberes. Diante dessa afirmação, é possível compreender que o enfermeiro cumpre o seu papel de articulador e integrador, reconhecido como um profissional que se comunica bem com a equipe multiprofissional, considerando que atua como elo entre essa equipe e os pacientes, seus principais focos da assistência. Assim, tanto o enfermeiro quanto à equipe da qual faz parte, tornam sua assistência dinâmica também. ${ }^{17-18}$

Portanto, deve-se ter em mente que a metodologia científica é um dos valores de grande importância para o agir profissional do enfermeiro, uma vez que lhe confere segurança na tomada de decisões relacionadas à nutriz e ao concepto, à sua equipe e às atividades administrativas da unidade. Isto se reflete positivamente na equipe de enfermagem, que tem o enfermeiro como seu condutor. Assim, a iniciativa para assumir condutas e atitudes está intimamente relacionada ao conhecimento que o profissional possui, pois este dá aos enfermeiros a certeza de estarem agindo da maneira correta e adequada. ${ }^{10,19}$

A aplicação da SAE torna o processo de cuidar em enfermagem mais amplo, permitindo um planejamento ainda mais individualizado e holístico, mantendo o conhecimento científico como âncora deste cuidado, desde que observadas todas as suas etapas. Contudo, os depoimentos não demonstraram um saber consolidado das participantes a respeito, tanto assim que consideraram a SAE uma questão de orientação individualizada - "cuidado individualizado", e até mesmo criterioso e sequencial, mas não apontaram como se constitui esse cuidado individualizado, ou ainda como ocorre esse acompanhamento sequencial. ${ }^{18,20-22}$

A utilização parcial da SAE pelos profissionais deve-se não só ao distanciamento entre a teoria e a prática mas, principalmente, por não haver uma preocupação maior com a qualidade da assistência, e sim com a demanda do serviço, ${ }^{6}$ o que não se justifica porque o cuidado é o fundamento da enfermagem e a SAE, aplicada na prática cotidiana do enfermeiro, possibilitaria que essa qualidade assistencial favorecesse a atuação do enfermeiro como difusor do conhecimento voltado para um cuidado qualificado. ${ }^{23-24}$

Apesar da SAE oferecer ao enfermeiro a possibilidade de organizar o seu trabalho com base em uma filosofia e um método que priorizam a individualidade do cuidado, os profissionais enfrentam adversidades para sua implementação.

Por outro lado, observam-se entre os motivos alegados para a sua não implementação, a falta de tempo, falta do conhecimento teórico de exercício prático e de recursos, além da organização de espaços para discussão da temática, desde a graduação. ${ }^{23}$ 


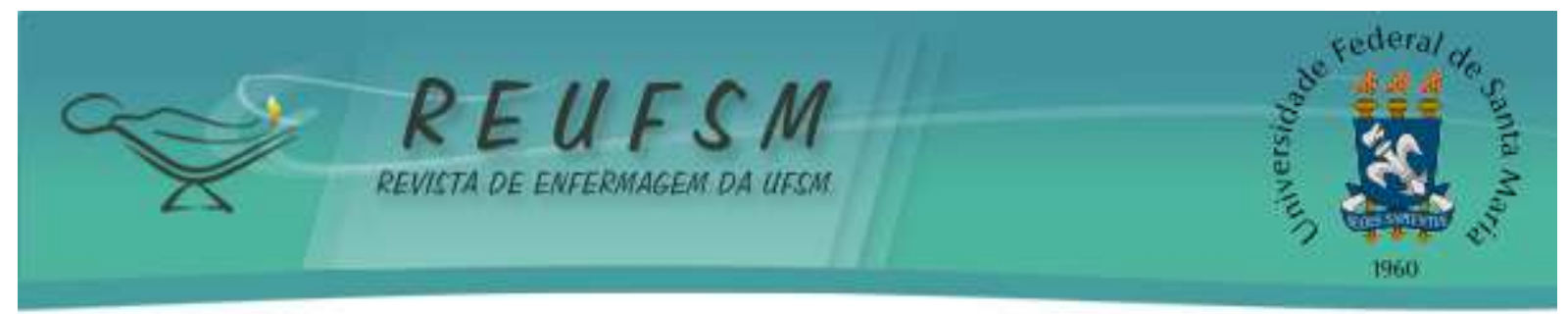

O Manual do BLH é considerado a "Bíblia" do Banco de Leite Humano. Essa frase resume muito bem a percepção das enfermeiras atuantes nos BLH. As enfermeiras, direta ou indiretamente, afirmaram que seguem as orientações prescritas nesse Manual. Considerando, porém, que o trabalho do enfermeiro deve estar embasado em uma metodologia científica ${ }^{8}$, a apropriação do conteúdo do citado Manual não deve ser a única fonte de suporte para a atuação do enfermeiro.

Nesse contexto, ao priorizar os POP, o enfermeiro pressupõe uma mecanização das ações de enfermagem. As ações sistematizadas e a aplicação do Processo de Enfermagem deixam de acontecer à luz das orientações contidas no Manual do BLH. A utilização dos POP, se agregado aos saberes dos enfermeiros, possibilita qualificar ainda mais as ações da enfermagem junto ao binômio mãe/bebê e as relações sociais/psicológicas/afetivas que envolvem esses participantes.

O Manual do BLH, assim como outros documentos normativos, confirma ser resultante de investigações e experiências acumuladas ao longo da existência dos BLH, mas também, são orientações das políticas públicas de saúde. Todavia, o que se analisa é a possibilidade de ampliar as ações dos enfermeiros que estão atuando nos BLH, ou que nele irão assumir uma função ao longo de sua atuação profissional, considerando que o referido manual possui orientações de ações padronizadas que podem e devem ser exploradas pelo enfermeiro para aperfeiçoar e elevar sua contribuição nesse espaço de transdisciplinaridade. ${ }^{3,25}$

O enfermeiro é reconhecido como um agente de transformação e sendo assim, sua participação em treinamentos e transmissão de novos saberes aos novos enfermeiros torna-se premente no exercício da enfermagem. ${ }^{19}$

É importante lembrar que as funções do profissional enfermeiro vinculam-se a três áreas ou campos de ação distintos, a saber: Área específica: assistir o ser humano no atendimento de suas necessidades básicas e torná-lo independente desta assistência, quando possível, pelo ensino do autocuidado; Área de interdependência ou de colaboração: a sua atividade na equipe de saúde nos aspectos de manutenção, promoção e recuperação da saúde; e Área social: dentro de sua atuação como um profissional a serviço da sociedade, função de pesquisa, ensino, administração, responsabilidade legal e de participação na associação de classe. ${ }^{20-21}$

O reconhecimento do papel do enfermeiro só é alcançado quando este utiliza um processo que dá transparência e fluidez ao seu trabalho. Isto ocorre quando ele se coloca em evidência como 'ponte' para os demais saberes e tudo se concretiza quando, junto ao paciente, busca compreender seu estado biológico, psicológico, emocional, espiritual e social, valendo- 


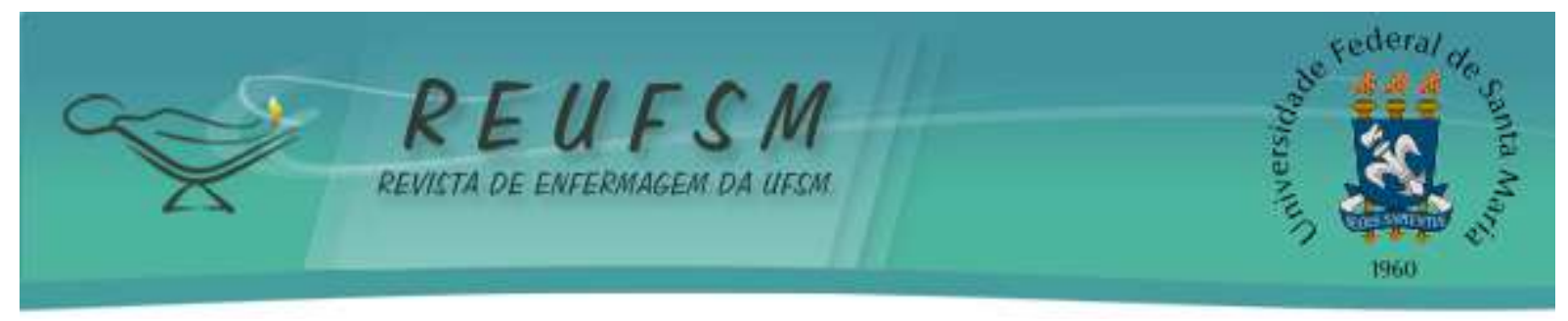

se de um instrumento sistematizado, um processo que utiliza um método, tendo assim a capacidade de colaborar com os demais profissionais que intervêm nesse mesmo paciente como uma fonte de informações preciosas para facilitar intervenções outras que sejam necessárias ante o quadro apresentado por esse paciente, foco do seu cuidado. ${ }^{21}$

\section{CONCLUSÃO}

A Sistematização da Assistência de Enfermagem no espaço do Banco de Leite Humano e a sua articulação à luz de suas normas técnicas podem ser consideradas fragmentadas, ficam em segundo plano quando expostas aos conhecimentos e prescrições contidas no manual técnico e normas do BLH.

Paralelo a isso, constata-se a necessidade de se implementar a SAE no espaço do Banco de Leite Humano, tendo em vista a perspectiva de se ampliar as possibilidades de trabalho neste espaço, bem como qualificar os trabalhos dos enfermeiros ao utilizar a SAE nas ações de atenção ao binômio mamãe/bebê. O trabalho desenvolvido no espaço do BLH pode usufruir de alguns aprimoramentos a partir da implementação da SAE, tendo em vista que sistematizar traz benefícios tanto ao paciente quanto à própria enfermagem, contribuindo para o efetivo trabalho desenvolvido pela equipe que atua nesse espaço especializado, acarretando confiabilidade e segurança no processo instituído.

Sabe-se que sistematizar depende de reconhecer as especificidades do trabalho da enfermagem e a importância de um arcabouço teórico acumulado ao longo da existência dessa profissão, assim como a necessidade de se continuar a produzir novos saberes que sustentem a qualidade da atenção à saúde/doença da população. Nesse sentido, a SAE no BLH implica na conscientização de saberes indispensáveis à prática dos enfermeiros. Para além, desdobra-se em atitudes e procedimentos que qualificam o atendimento à paciente/nutriz e fomentam ações de consolidação das políticas públicas de apoio ao aleitamento materno.

As enfermeiras em suas rotinas contemplam a orientação da equipe e a distribuição de tarefas, o acompanhamento das atividades administrativas, a construção e checagem de novos instrumentos, POP, para qualificar os serviços do BLH, a orientação à paciente/cliente, a atuação na assistência e gerenciar a formação continuada dos profissionais da equipe. Suscita, então, a necessidade de se elaborar estratégias que articulem a SAE e os POP nos BLH, fato que pode ampliar as ações da equipe atuante nesse espaço especializado. 


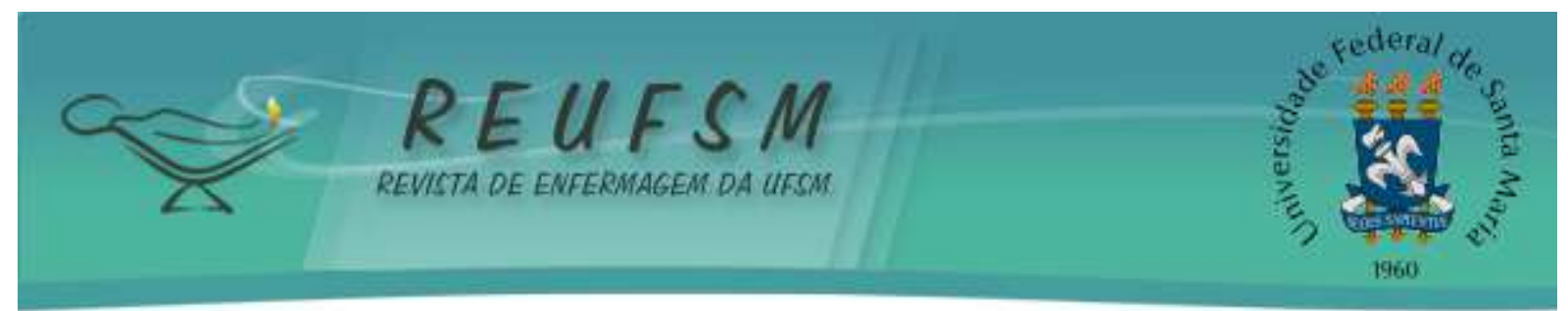

As políticas públicas no Brasil que envolvem os Bancos de Leite Humano podem suscitar investimentos na capacitação dos enfermeiros, mediante elaboração de instrumento de coleta de dados e acompanhamento de paciente, utilização dos POP para aproximação com este instrumento metodológico, aliado ao conhecimento das teorias de enfermagem.

A observação e apreciação das práticas desses profissionais no BLH podem revelar nuances que este trabalho não conseguiu expressar. Assim, faz-se oportuno indicar também, pesquisas que auxiliem a compreender a participação dos enfermeiros nas normativas do BLH, histórico quanto à elaboração/efetivação/validação dos manuais, das normas, bem como a colaboração desse profissional com os saberes sistematizados que organizam a prática cotidiana nos BLH.

Não foram encontrados estudos, de modo satisfatório, que auxiliassem na discussão da temática em foco, sendo este um fator limitante da pesquisa. Além disso, são indicadas novas pesquisas sobre as práticas dos enfermeiros nos BLH e a articulação da SAE com o manual de normas técnicas da ANVISA, uma vez que a análise em foco das falas das entrevistadas pode expressar um recorte dessa realidade precária, no campo da pesquisa.

\section{REFERÊNCIAS}

1. Vieczoerek, AL; Wolff LDG. Evaluation of human milk banks in Paraná - Br: a comparative study. Online Braz J Nurs [Internet]. 2012 [acesso em 2014 out 21];11(1):1-11. Disponível em: http://www.objnursing.uff.br/index.php/nursing/article/view/3562/pdf.

2. Centro de Referência Nacional para Bancos de Leite Humano - Instituto Fernandes Figueira. Normas Técnicas para Bancos de Leite Humano. Rio de Janeiro: FIOCRUZ/IFFBLH; 2006.

3. Brasil. Agência Nacional de Vigilância Sanitária (Anvisa). Banco de Leite Humano: funcionamento, prevenção e controle de riscos. Brasília: Anvisa; 2008.

4. Moura GMSS, Inchauspe JAF, Dall'Agnol CM, Magalhães AMM, Hoffmeister LV. Expectativas da equipe de enfermagem em relação à liderança. Acta Paul Enferm. 2013;26(2):198-204.

5. Varela GC, de Azevedo Fernandes SC. Conhecimentos e práticas sobre a sistematização da assistência de enfermagem na estratégia saúde da família. Cogitare Enferm. 2013;18(1):124-30.

6. Santos FO, Montezeli JH, Peres AM. Autonomia profissional e sistematização da assistência de enfermagem: percepção de enfermeiros. REME [Internet]. 2012 [acesso em 2016 ago 12];16(2):251-7. Disponível em: http://www.reme.org.br/artigo/detalhes/526.

7. Santos RB, Ramos KS. Sistematização da assistência de enfermagem em Centro Obstétrico. Rev Bras Enferm [Internet]. 2012 [acesso em 2016 ago 12];65(1):13-8. Disponível em: http://dx.doi.org/10.1590/S0034-71672012000100002. 


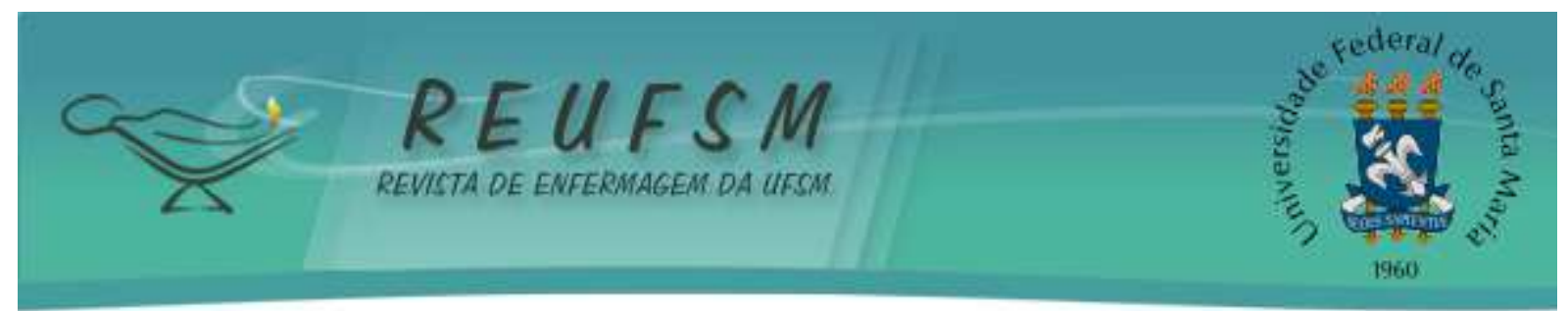

8. Conselho Federal de Enfermagem. Resolução COFEN n॰ 358 de 15 de outubro de 2009. Dispõe sobre a Sistematização da Assistência de Enfermagem e a implementação do Processo de Enfermagem em ambientes, públicos ou privados, em que ocorre o cuidado profissional de Enfermagem, e dá outras providências. Rio de Janeiro: Conselho Federal de Enfermagem; 2009.

9. Figueiredo MED, Santos SR, Oliveira AMM, Leite NS, Morais JMD, Duarte ACP. Sistematização da Assistência de Enfermagem: Percepção de Enfermeiros de um Hospital Escola. Rev Enferm UFPE [Internet]. 2013 [acesso em 2016 ago 12];7(12):6981-8. Disponível em: http://www.revista.ufpe.br/revistaenfermagem/index.php/revista/article/view/4828/pdf_4216.

10. Maria MA, Quadros FAA, Grassi MFO. Sistematização da assistência de enfermagem em serviços de urgência e emergência: viabilidade de implantação. Rev Bras Enferm [Internet]. 2012 [acesso em 2016 ago 12];65(2):297-303. Disponível em: http://dx.doi.org/10.1590/S0034-71672012000200015.

11. Baptista SS, Alves VH, Souza RDMP, Rodrigues DP, Cruz AFN, Branco MBLR. Manejo clínico da amamentação: atuação do enfermeiro na Unidade de Terapia Intensiva Neonatal. Rev Enferm UFSM. 2015;5(1):23-31.

12. Roa AC, Silva FRB. A Fiocruz como ator da política externa brasileira no contexto da comunidade dos países de língua portuguesa: uma história revelada. Hist Cienc Saude Manguinhos [Internet]. 2015 [acesso em 2017 maio 23];22(1):153-69. Disponível em: http://www.scielo.br/pdf/hcsm/v22n1/pt_0104-5970-hcsm-22-01-00153.pdf.

13. Hages F, Alemseged F, Balcha F, Berhe S, Aregay A. Application of nursing process and its affecting factors among nurses working in mekelle zone Hospitals, Northern Ethiopia. Nurs Res Pract [Internet]. 2014 [acesso em 2017 maio 23];2014(1):1-9. Disponível em: http://www.hindawi.com/journals/nrp/2014/675212/.

14. Zamanzadeh V, Valizadeh L, Tabrizi FJ, Behshid M, Lotfi M. Challenges associated with the implementation of the nursing process: A systematic review. Iran J Nurs Midwifery Res [Internet]. 2015 [acesso em 2017 maio 23];20(4):411-9. Disponível em: http://dx.doi.org/10.4103/1735-9066.161002.

15. Minayo MCS. O desafio do conhecimento: pesquisa qualitativa em saúde. $13^{a}$ ed. São Paulo: Hucitec; 2013.

16. Bardin L. Análise de conteúdo. Lisboa: Edições 70; 2011.

17. Duarte GM, Alves MS. Trabalho em equipe/proximidade do paciente: elementos da práxis de enfermeiras na terapia intensiva. Rev Enferm UFSM. 2014;4(1):144-51.

18. Fernández-Sola C, Granero-Molina J, Mollinedo-Mallea J, Gonzales MHP, AguileraManrique G, Ponce ML. Desarrollo y validación de un instrumento para la evaluación inicial de enfermeira. Rev Esc Enferm USP [Internet]. 2012 [acesso em 2016 ago 12];46(6):1415-22. Disponível em: http://www.scielo.br/pdf/reeusp/v46n6/19.pdf.

19. Zanetti ML. Prática avançada de enfermagem: estratégias para formação e construção do conhecimento. Rev Latinoam Enferm [Internet]. 2015 [acesso em 2017 fev 05];23(5):779-8. Disponível em: http://www.scielo.br/pdf/rlae/v23n5/pt_0104-1169-rlae-23-05-00779.pdf. 


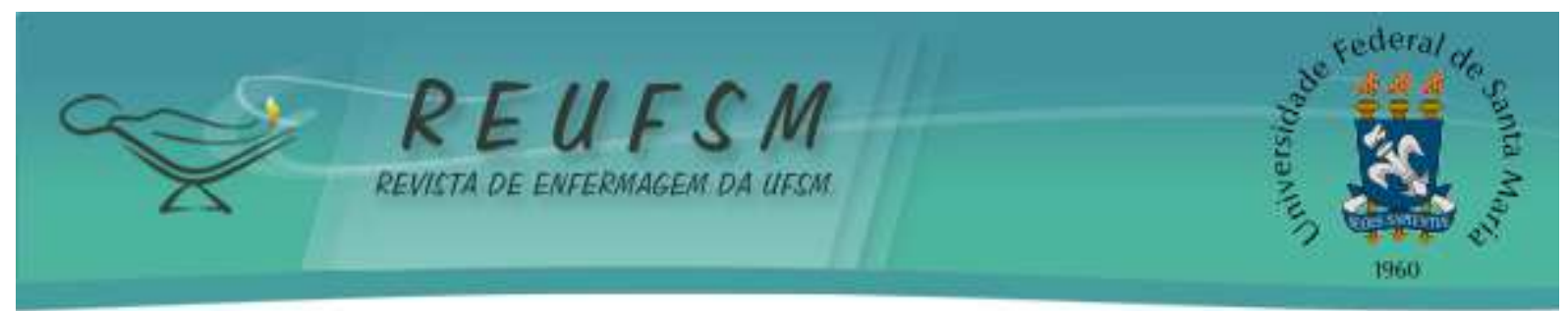

20. Lima MM, Kloh D, Canever BP, Reibnitz KS, Amestoy SC, Prado ML. Integralidade na formação do enfermeiro: possibilidades de aproximação com os pensamentos de Freire. Saúde Transform Soc [Internet]. 2013 [acesso em 2017 fev 20];4(4),3-8. Disponível em: http://pepsic.bvsalud.org/scielo.php?script=sci_arttext\&pid=S2178-

$70852013000400003 \& \operatorname{lng}=\mathrm{pt} \& \operatorname{lng}=\mathrm{p}$.

21. Backes DS, Backes MS, Erdmann AL, Büscher A. O papel profissional do enfermeiro no Sistema Único de Saúde: da saúde comunitária à estratégia de saúde da família. Ciênc saúde Colet [Internet]. 2012 jan [acesso em 2017 fev 20];17(1):223-30. Disponível em: http://www.scielo.br/scielo.php?script=sci_arttext\&pid=S1413$81232012000100024 \& \operatorname{lng}=\mathrm{en}$.

22. Mangueira SO, Lima JTS, Costa SLA, Nóbrega MML, Lopes MVO. Implantação da sistematização da assistência de enfermagem: opinião de uma equipe de enfermagem hospitalar. Enferm Foco [Internet]. 2012 [acesso em 2017 fev 04];3(3):135-8. Disponível em: http://revista.portalcofen.gov.br/index.php/enfermagem/article/viewFile/298/160.

23. Figueiredo PP, Lunardi Filho WD, Silveira RS, Fonseca AD. A não implementação do processo de enfermagem: reflexão apoiada em conceitos de Deleuze e Guattari. Texto \& Contexto Enferm. 2014;23(4):1136-44.

24. Souza NR, Costa BMB, Carneiro DCDF, Barbosa HSC, Santos ICRV. Systematization of nursing care: difficulties referred by nurses of a university hospital. Rev Enferm UFPE [Internet]. 2015 [acesso em 2017 fev 20];9(3):7104-10. Disponível em: http://www.revista.ufpe.br/revistaenfermagem/index.php/revista/article/view/6337/pdf_7399.

25. Branco MBLR, Alves VH, Rodrigues DP, Souza RDMP, Lopes FO, Marinho TF. Proteção e apoio ao aleitamento materno: uma contribuição do banco de leite. Rev Pesqui Cuid Fundam [Internet]. 2016 abr/jun [acesso em 2017 fev 05];8(2):4300-12. Disponível em: http://www.seer.unirio.br/index.php/cuidadofundamental/article/view/4357/pdf_1876.

Data de submissão: 26/01/2017

Data de aceite: 10/08/2017

Contato do autor principal: Giovanna Rosario Soanno Marchiori

Endereço postal: Av. Santa Leopoldina, 840 - Bairro Coqueiral de Itaparica, Vila Velha/ES, CEP 29102-041. E-mail: giovannasoanno@gmail 